\title{
HILBERT INEQUALITY AND GAUSSIAN HYPERGEOMETRIC FUNCTIONS
}

\author{
MARIO KRNIĆ
}

Abstract. By using the integral representation of Gaussian hypergeometric function, we obtain Hilbert type inequalities with some fractional kernels and non-conjugate parameters. Such inequalities include the constant factors expressed in terms of hypergeometric functions. Further, we obtain the best possible constants for some general cases, in conjugate case.

Mathematics subject classification (2000): 26 D15.

Keywords and phrases: Inequalities, Hilbert type inequality, Hardy-Hilbert type inequality, equivalent inequalities, non-conjugate parameters, beta function, gamma function, the best possible constant, hypergeometric function, Gaussian hypergeometric function, Pfaff's reflection law, Euler identity.

\section{REFERENCES}

[1] M.Abramowitz, I.A.STEgun, Handbook of mathematical function with formulae, graphs and mathematical tables, National Bureau of Standards, Applied Math. Series 55, 4th printing, Washington, 1965.

[2] F.F. Bons ALL, Inequalities with non-conjugate parameters, Quart. Journ. Math. Oxford, 2, 2 (1951), $135-150$.

[3] R.L. Graham, D.E. Knuth, D. Patashnik, Concrete mathematics- A Foundation for Computer Science, Addison-Wesley Publishing Company, 1989.

[4] G.H. Hardy, J.E. Littlewood and G. Pólya, Inequalities, Cambridge Univ. Press, Cambridge, 1952.

[5] M. Krnić, J. PeČArić, Hilbert's inequalities and their reverses, Publ. Math. Debrecen, 67, 3-4 (2005), 315-331.

[6] M. Krnić, J. PeČArić, General Hilbert's and Hardy's inequalities, Math. Inequal. Appl., 8, 1 (2005), 29-52.

[7] M. KRnić, J. PEČARIĆ, Extension of Hilbert's Inequality, J. Math. Anal. Appl., 324 (2006), 150-160.

[8] V. Levin, On the Two Parameter Extension and Analogue of Hilbert's Inequality, J. London Math. Soc., 11 (1936), 119-124.

[9] D.S. Mitrinović, J.E. PeČArić And A.M. Fink, Classical and new inequalities in analysis, Kluwer Academic Publishers, Dordrecht/Boston/London, 1993.

[10] B. YAng, On Hilberts integral inequality, J. Math. Anal. Appl., 220 (1998), 778-785. 